\title{
A prospective comparison of nursing home acquired pneumonia with community acquired pneumonia
}

\author{
W.S. Lim, J.T. Macfarlane
}

A prospective comparison of nursing home acquired pneumonia with community acquired pneumonia. W.S. Lim, J.T. Macfarlane. (C) ERS Journals Ltd 2001.

ABSTRACT: Nursing home acquired pneumonia (NHAP) is thought to be clinically distinct from community acquired pneumonia (CAP). This observation, based on studies conducted mainly in North America, may not be relevant in countries with a different healthcare system.

The authors describe an 18-month prospective cohort study of 437 patients admitted to hospital with CAP, $40(9 \%)$ of whom came from nursing homes. Detailed microbiological tests were performed in a subset of patients over 12 months.

Patients with NHAP were less likely to have a productive cough (odds ratio (OR) $0.4, p=0.02)$ or pleuritic pain (OR $0.1, p=0.03$ ), but they were more likely to be confused (OR 2.6, $p<0.001)$. They had poorer functional status $(p<0.001)$ and more severe disease $(\mathrm{p}=0.03)$. Mortality was higher compared to CAP $(53 \%$ versus $13 \%)$, but this was mainly explained by prior functional status (OR 0.5, after adjustment for functional status). Pathogens were identified in $68 \%$ of 22 NHAP and $80 \%$ of 44 matched CAP patients. Streptococcus pneumoniae was the most common (55\% NHAP, 43\% CAP). Atypical pathogens, enteric Gram negative bacilli and Staphylococcus aureus were uncommon.

In conclusion, differences in functional status accounted for the increased mortality in nursing home acquired pneumonia compared to community acquired pneumonia. The pathogens implicated were similar. No grounds for a difference in choice of empirical antibiotics were apparent.

Eur Respir J 2001; 18: 362-368.
Respiratory Medicine, Nottingham City Hospital, Nottingham, UK.

Correspondence: J.T. Macfarlane, Respiratory Medicine, Nottingham City Hospital, Hucknall Road, Nottingham NG5 1PB, UK

Fax: 441159627723

Keywords: Aetiology cohort study

community acquired pneumonia

nursing home acquired pneumonia

Received: January 122001

Accepted after revision March 202001

This study was supported by a research grant from Hoechst Marion Roussel.
Nursing home acquired pneumonia (NHAP) is the leading cause of death among long-term care patients and one of the most common causes of transfer to hospital, where it accounts for $2-18 \%$ of patients hospitalized for pneumonia [1-3].

NHAP has been considered to differ from community acquired pneumonia (CAP), a higher mortality and also different aetiology, with some studies reporting infection by enteric Gram negative bacilli (EGNB) and Staphylococcus aureus to be much more common in NHAP [4-6]. When compared to elderly persons with CAP, patients with NHAP have been found to have increased levels of dependency, cerebrovascular disease, disease severity and mortality. No differences in processes of care, including time from hospital admission to antibiotic administration have been reported; although rates of mechanical ventilation were lower in patients with NHAP in one study $[4,7]$. Based on these findings, different antibiotic strategies have been advocated for patients with NHAP [8]. However, most of the published studies have come from North America, where nursing home facilities vary markedly in terms of diagnostic and treatment capabilities and management policies [9-11]. Thus, it is unlikely that they represent the situation in the UK and the rest of Europe, where no studies of NHAP have been reported.
The present prospective cohort study compares the clinical features and outcomes of patients admitted to hospital with NHAP and CAP in the UK.

\section{Methods}

The present study was approved by the hospital ethics committee and was conducted at Nottingham City Hospital (NCH) October 4, 1998-March 31, 2000. This hospital shares equally with another hospital in all emergency adult hospital admissions in the Nottingham district. There are no other acute care facilities for elderly patients requiring hospitalization in Nottingham. Consecutive adults aged $\geqslant 16$ yrs admitted with a diagnosis of CAP were studied prospectively and standardized clinical data and investigations were obtained on admission. CAP was defined as the presence of an acute illness of $\leqslant 21$ days duration with the following. 1) Features of a lower respiratory tract infection including two or more of: new or increasing cough, sputum production, shortness of breath, wheeze, chest pain, new focal or diffuse signs on chest examination; and one or more constitutional symptoms including fever, confusion, sweating, headaches, aches and pains, sore throat or coryza. 2) Radiographic shadowing on an admission 
chest radiograph consistent with infection and which is neither pre-existing nor of another known cause. 3) Treatment with antibiotics for pneumonia by the attending physician. Patients were excluded where pneumonia was not the primary cause for hospital admission, an expected terminal event or distal to bronchial obstruction (e.g. from lung cancer). Patients with tuberculosis and human immunodeficiency virus (HIV) were excluded, as were patients who had been in hospital within the previous 10 days, were immunocompromised (received chemotherapeutic agents within the $\geqslant 6$ months than the equivalent of prednisolone $10 \mathrm{mg}$ daily for $\geqslant 3$ months prior to admission) or had previously been entered in the study. Comorbid illness was defined as the presence of any of the following conditions for which the patient was under active medical supervision or was receiving treatment at the time of hospital admission: chronic lung disease, cardiac disease (ischaemic heart disease, cardiac failure, hypertension, atrial fibrillation), cerebrovascular disease (including previous transient ischaemic attacks), cognitive impairment, diabetes mellitus, chronic liver disease, chronic renal disease and inflammatory rheumatological disorders (excluding osteoarthiritis). Mental confusion was defined as an Abbreviated Mental Test Score of $\leqslant 8$. Severity of illness on admission was assessed using the modified British Thoracic Society (mBTS) prediction rule and the Pneumonia Severity Index (PSI) [12, 13]. Patients admitted from nursing homes with pneumonia were considered to have NHAP. All patients were seen within $24 \mathrm{~h}$ of admission by a study investigator to confirm study entry criteria and informed consent. Premorbid functional status in all patients admitted after October 3, 1999 was determined by patient history using the European Cooperative Oncology Group (ECOG) functional status scale and the Barthel Index. Patient reported influenza and pneumococcal vaccination status was also recorded.

\section{Investigating aetiology}

Comprehensive microbiological investigations were conducted on all patients admitted with CAP between October 4, 1998-October 3, 1999. Full details are reported elsewhere [14]. Briefly, samples of sputum, blood and urine were collected within $24 \mathrm{~h}$ of admission and a further sample for convalescent serology was collected 4-6 weeks after discharge. Investigations performed included sputum culture, blood culture, complement fixation tests for antibodies to mycoplasma, chlamydia, Q fever, influenza $\mathrm{A}$ and $\mathrm{B}$, respiratory syncytial virus and adenoviruses, immunofluorescent antibody testing (using formalized yolk sac antigen of Legionella pneumophilia serogroup 1) for antibodies to Legionella, serological tests for Haemophilus influenzae, Moraxella catarrhalis, Streptococcus pneumoniae and Chlamydia pneumoniae, pneumococcal antigen detection in sputum by countercurrent immunoelectrophoresis (CIE) and pneumococcal and Legionella antigen detection in urine by commercial kits (Binax-NOW; Binax, Inc., Portland, ME, USA). In order to compare the spectrum of microbial pathogens implicated in NHAP and CAP taking into account seasonality and an expected higher frequency of atypical pathogens in patients aged <75 yrs [14], patients with NHAP admitted October 4, 1998-October 3, 1999 were matched with two controls with CAP. Controls were drawn from patients admitted with CAP within 2 months of the case and matched for age (within 5 yrs) and sex.

\section{Statistical analysis}

Chi-squared tests were used to compare categorical variables. Multivariate analysis was performed by stepwise logistic regression. Adjustment for age was conducted a priori in all analyses. Adjustment for disease severity was performed using the PSI as a binary variable (threshold value of 90 to distinguish between low and high risk groups) and the mBTS rule. Adjustment for functional status was performed using an ECOG level of 1 or 2 to denote good performance status compared to levels 3 and 4, and a Barthel score of $\leqslant 15$ to describe limited self-care ability [15]. Results are expressed as age-adjusted odds ratios (ORs) with $95 \%$ confidence intervals (CIs) and p-values, taking $\mathrm{p}<0.05$ as the level of statistical significance. Duration of intravenous antibiotic therapy was calculated according to "i.v. antibiotic days" (1 "i.v. antibiotic day" $=1$ day's equivalent standard $i . v$. doses e.g. three doses of $i . v$. cefuroxime or four doses of $i . v$. erythromycin).

\section{Results}

Of 437 patients admitted with CAP, 40 (9\%) had NHAP. Mean \pm sD age of patients with NHAP was $80.6 \pm 9.6$ yrs compared to $65.5 \pm 19$ yrs for patients with CAP $(\mathrm{p}<0.001)$. There was a trend for more patients with NHAP to have one or more comorbid illnesses $(90 \%)$, compared to patients with CAP $(75 \%)$ $(\mathrm{p}=0.06)$. Underlying dementia (OR 8.2, 95\% CI $3.4-19.5, \mathrm{p}<0.001)$ and cerebrovascular disease (OR $3.7,95 \%$ CI $1.7-7.9, \mathrm{p}<0.001)$ were significantly more common in patients with NHAP (table 1). Functional status, as measured in 154 patients using the Barthel index and in 149 patients using the ECOG performance status scale, was significantly lower in patients with NHAP $(\mathrm{p}<0.001)$.

\section{Symptoms at presentation}

There was no difference in symptoms at presentation between the three groups except that patients with NHAP were less likely to have a productive cough (OR 0.4, 95\% CI 0.2-0.9, p=0.02) and pleuritic chest pain (OR $0.1,95 \%$ CI $0.01-0.8, \mathrm{p}=0.03$ ) (table 2). The proportions of patients reporting fever or presenting "off legs" were similar. On admission to hospital, patients with NHAP had lower systolic blood pressure, higher urea and lower serum albumin levels than patients with CAP (table 3). Mental 
Table 1.-Patient characteristics and outcomes

\begin{tabular}{|c|c|c|c|c|}
\hline & NHAP & CAP aged $\geqslant 65$ yrs & All CAP & p-value $\#$ \\
\hline Subjects $\mathrm{n}$ & 40 & 236 & 397 & \\
\hline Age yrs & $80.6 \pm 9.6$ & $79 \pm 8$ & $65.5 \pm 19.4$ & $<0.001^{\top}$ \\
\hline Range yrs & 55-97 & $65-100$ & $17-100$ & \\
\hline Median yrs & 81.5 & & 70 & \\
\hline Sex M & $22(55)$ & $199(50)$ & $199(50)$ & 0.8 \\
\hline \multicolumn{5}{|l|}{ Comorbid illnesses } \\
\hline 1 & $18(42)$ & $92(39)$ & $148(37)$ & \\
\hline 2 & $8(20)$ & $68(29)$ & $94(24)$ & \\
\hline$\geqslant 3$ & $9(22.5)$ & $48(20)$ & $51(13)$ & \\
\hline None & $5(10)$ & $28(12)$ & $103(26)$ & 0.9 \\
\hline Cancer & $2(5)$ & $19(2)$ & $21(5.3)$ & 0.5 \\
\hline Cerebrovascular disease & $14(35) *$ & $33(14)^{*}$ & $36(9.1)$ & $<0.001$ \\
\hline Congestive cardiac failure & $3(7.5)$ & $22(9)$ & $22(5.5)$ & 0.7 \\
\hline Ischaemic heart disease & $4(10)$ & $47(20)$ & $55(14)$ & 0.2 \\
\hline Chronic obstructive pulmonary disease & $8(20)$ & $73(31)$ & $89(22)$ & 0.4 \\
\hline Diabetes mellitus & $2(5)$ & $25(11)$ & $33(8)$ & 0.4 \\
\hline Dementia & $14(35) *$ & $18(8)^{*}$ & $33(8)$ & $<0.001$ \\
\hline Prior antibiotics & $18(45)$ & $97(41)$ & 145(37) & 0.6 \\
\hline Influenza vaccination in last yr & $15(38)$ & $101(43)$ & $132(33)$ & 0.9 \\
\hline Pneumococcal vaccination in last 10 yrs & $3(7.5)^{*}$ & $49(21)^{*}$ & $63(16)$ & 0.1 \\
\hline ECOG $(1-4)$ & $3 \pm 1 *$ & $1.6 \pm 1.2 *$ & $1.2 \pm 1.2$ & $<0.001$ \\
\hline Barthel index $(0-20)$ & $8.9 \pm 6.7 *$ & $16 \pm 5.1 *$ & $17 \pm 5.1$ & $<0.001$ \\
\hline
\end{tabular}

Data are presented as $\mathrm{n}(\%)$ and mean \pm SD unless otherwise stated. NHAP: nursing home acquired pneumonia. CAP: community acquired pneumonia; M: male; ECOG: European Cooperative Oncology Group. \#: age-adjusted: comparing NHAP with all CAP; ${ }^{\prime}$ : not age-adjusted; *: statistically significant difference $(\mathrm{p}<0.05$, not age-adjusted).

confusion was detected more often in patients with NHAP (OR 3.9, 95\% CI 1.9-7.9, p<0.001).

Aetiology. Detailed microbiological tests were performed in 22 patients with NHAP and 44 controls (CAP) over one year. In patients with NHAP, samples of sputum were obtained in $7(32 \%)$, blood for culture in $16(73 \%)$, serum for serology in $17(77 \%)$ and urine for antigen testing in $13(59 \%)$. Corresponding figures for controls were $28(64 \%), 36(82 \%), 43(98 \%)$ and 39 $(89 \%)$. A pathogen was detected in $50(77 \%)$ of 66 patients, most commonly S. pneumoniae; in 12 (55\%) cases and $19(43 \%)$ controls $(\mathrm{p}=0.38)$. Apart from C. pneumoniae (four cases, seven controls), atypical pathogens were rarely found (table 4). No EGNB were detected and $S$. aureus was detected in one control.
Influenza A infection was found in $32 \%$ of controls and no cases. The influenza immunization rate in the 66 patients who had detailed microbiological tests was $45 \%$ (10 patients) for cases and 36\% (16 patients) for controls.

\section{Treatment}

Patients with NHAP were more likely to receive empirical intravenous antibiotics as initial therapy (93\% vs 74\%, OR 3.9, 95\% CI 1.1-13.1, $\mathrm{p}=0.03)$, however, for patients receiving i.v. antibiotics, the mean duration of therapy was no different between groups (table 5). Intravenous cefuroxime was the most commonly used antibiotic $(85 \%$ NHAP and

Table 2. - Symptoms at presentation

\begin{tabular}{|c|c|c|c|c|}
\hline & NHAP & CAP aged $\geqslant 65$ yrs & All CAP & p-value ${ }^{\#}$ \\
\hline Subjects n & 40 & 236 & 397 & \\
\hline \multicolumn{5}{|l|}{ Respiratory } \\
\hline Cough & $25(63)$ & $171(72)$ & $297(75)$ & 0.20 \\
\hline Sputum production & $12(30)^{*}$ & $124(53)^{*}$ & $209(53)$ & 0.02 \\
\hline Dyspnoea & $23(58) *$ & $175(74)^{*}$ & $259(65)$ & 0.07 \\
\hline Haemoptysis & $2(5)$ & $7(3)$ & $15(4)$ & 0.40 \\
\hline Pleuritic pain & $1(2.5)^{*}$ & $41(17)^{*}$ & $122(31)$ & 0.03 \\
\hline \multicolumn{5}{|l|}{ General } \\
\hline Fever & $10(25)^{*}$ & $29(12)^{*}$ & $81(20)$ & 0.10 \\
\hline Confusion & $12(30)^{*}$ & $29(12)^{*}$ & $49(12)$ & 0.01 \\
\hline "Off legs" & $8(20)$ & $42(18)$ & 47 (12) & 1.00 \\
\hline Diarrhoea & $2(5)$ & $6(3)$ & $22(6)$ & 0.60 \\
\hline Flu-like illness & $2(5)$ & $14(6)$ & $41(10.3)$ & 0.60 \\
\hline
\end{tabular}

Data are presented as n (\%) unless otherwise stated. NHAP: nursing home acquired pneumonia; CAP: community acquired pneumonia. ${ }^{\#}$ : age-adjusted: comparing NHAP with all CAP. *: statistically significant difference ( $\mathrm{p}<0.05$, not age-adjusted). 
Table 3. - Clinical findings on presentation

\begin{tabular}{|c|c|c|c|c|}
\hline & NHAP & CAP aged $\geqslant 65$ yrs & All CAP & p-value \\
\hline Subjects n & 40 & 236 & 397 & \\
\hline Temperature ${ }^{\circ} \mathrm{C}$ & $34.9 \pm 8.4$ & $36.6 \pm 4.4$ & $36.7 \pm 4.7$ & 0.06 \\
\hline Pulse beats $\cdot \min ^{-1}$ & $106 \pm 25.4 *$ & $98 \pm 18^{*}$ & $100 \pm 20$ & 0.07 \\
\hline SBP $\mathrm{mmHg}$ & $123 \pm 26^{*}$ & $135 \pm 28 *$ & $131 \pm 26$ & 0.03 \\
\hline DBP $\mathrm{mmHg}$ & $75 \pm 15$ & $73 \pm 14$ & $73 \pm 14$ & 0.20 \\
\hline $\mathrm{RR} \min ^{-1}$ & $27 \pm 8$ & $27 \pm 8$ & $26 \pm 8$ & 0.50 \\
\hline $\mathrm{O}_{2}$ saturation $\%$ & $86.7 \pm 12$ & $87.4 \pm 15$ & $88.3 \pm 15$ & 0.80 \\
\hline Haemoglobin $\mathrm{g} \cdot \mathrm{dL}^{-1}$ & $12.4 \pm 1.9$ & $13.1 \pm 7.5$ & $12.9 \pm 1.9$ & 0.30 \\
\hline White cell count & $14 \pm 8.8$ & $13 \pm 5.8$ & $13 \pm 5.8$ & 0.20 \\
\hline Urea & $14.9 \pm 10.8^{*}$ & $10.5 \pm 11^{*}$ & $8.2 \pm 5.5$ & $<0.001$ \\
\hline Creatinine & $140 \pm 73.8$ & $127 \pm 71$ & $115 \pm 63$ & 0.20 \\
\hline Albumin & $29 \pm 5.1 *$ & $33 \pm 4.8^{*}$ & $33 \pm 5.2$ & $<0.001$ \\
\hline C-reactive protein & $200 \pm 136$ & $170 \pm 124$ & $187 \pm 136$ & 0.20 \\
\hline Glucose & $7.9 \pm 3.5$ & $7.3 \pm 4.2$ & $7.8 \pm 5.2$ & 0.80 \\
\hline Temperature $<37^{\circ} \mathrm{C}$ & $19(48)$ & $107(45)$ & $153(39)$ & 0.30 \\
\hline $\mathrm{RR} \geqslant 30 \mathrm{~min}^{-1}$ & $18(45)$ & $86(37)$ & $132(33)$ & 0.20 \\
\hline Confusion & $24(60)^{*}$ & $64(27)^{*}$ & $82(21)$ & $<0.001$ \\
\hline $\mathrm{DBP} \leqslant 60 \mathrm{mmHg}$ & $12(30)$ & $66(28)$ & $105(26)$ & 0.80 \\
\hline Urea $>7 \mathrm{mmol} \cdot \mathrm{L}^{-1}$ & $30(75)$ & $146(62)$ & $184(46)$ & 0.10 \\
\hline Albumin $<30 \mathrm{~g} \cdot \mathrm{dL}^{-1}$ & $18(45)^{*}$ & $47(20)^{*}$ & $83(21)$ & $<0.001$ \\
\hline Severe CAP, mBTS rule & $28(70)^{*}$ & $119(50)^{*}$ & $153(39)$ & 0.03 \\
\hline PSI score & $144 \pm 26 *$ & $110 \pm 30^{*}$ & $90 \pm 39$ & $<0.001$ \\
\hline Risk Class I & 0 & 0 & $54(14)$ & \\
\hline Risk Class II & 0 & $17(7)$ & 76 (19) & \\
\hline Risk Class III & $1(3)$ & $52(22)$ & 76 (19) & \\
\hline Risk Class IV & $13(32)$ & $113(48)$ & $130(33)$ & \\
\hline Risk Class V & $26(65)$ & $54(23)$ & $61(15)$ & \\
\hline
\end{tabular}

All data are presented as mean $\pm \mathrm{SD}$ and $\mathrm{n}(\%)$ unless otherwise stated. NHAP: nursing home acquired pneumonia; CAP: community acquired pneumonia; SBP: systolic blood pressure; DBP: diastolic blood pressure; RR: respiratory rate; mBTS: modified British Thoracic Society; PSI: Pneumonia Severity Index. \#: age-adjusted: comparing NHAP and all CAP. *: statistically significant difference ( $\mathrm{p}<0.05$, not age-adjusted).

$69 \%$ CAP), and it was given in combination with a macrolide in $17(43 \%)$ patients with NHAP compared to $169(43 \%)$ patients with CAP. Of the four NHAP and nine CAP patients with identified infection by atypical pathogens, two and four patients,

Table 4. - Microbial aetiology: based on detailed tests performed October 4, 1998-October 3, 1999. Cases include those with nursing home acquired pneumonia (NHAP). Controls are age- and sex-matched patients with community acquired pneumonia (CAP), not NHAP

\begin{tabular}{lcc}
\hline & Cases & Controls \\
\hline Subjects n & 22 & 44 \\
Pathogen detected & $15(68)$ & $35(80)$ \\
Bacterial & $13(59)$ & $24(55)$ \\
Atypical & $4(18)$ & $9(20)$ \\
Viral & $3(14)$ & $15(34)$ \\
Mixed & $5(23)$ & $14(32)$ \\
Streptococcus pneumoniae & $12(55)$ & $19(43)$ \\
Staphylococcus aureus & 0 & 1 \\
Moraxella catarrhalis & 1 & 1 \\
Haemophilus influenzae & 0 & $5(11)$ \\
Chlamydia pneumoniae & $4(18)$ & $7(16)$ \\
Chlamydia psittaci & 0 & 1 \\
Legionella sp. & 0 & 1 \\
Influenza A virus & 0 & $14(32)$ \\
Influenza B virus & 0 & 1 \\
Respiratory syncytial virus & $3(14)$ & 0 \\
\hline
\end{tabular}

Data are presented as n (\%) unless otherwise stated. respectively, received inadequate empirical antibiotics. All six patients survived.

No NHAP patients were was admitted to the intensive care unit (ICU) compared to $20(5 \%)$ CAP patients.

\section{Severity assessment and mortality}

NHAP was associated with more severe pneumonia, whether assessed according to the mBTS rule $(\mathrm{p}=0.03)$ or the PSI $(\mathrm{p}<0.001)$, and carried a higher inhospital mortality compared with CAP (53\% versus $13.4 \%$ ). The association of NHAP with increased mortality was significant after adjusting for age, ICU admission and disease severity (PSI) (OR 2.3 95\% CI 1.1-5.0. $\mathrm{p}=0.03$ ), but was not evident after adjustment for functional status (ECOG) (OR 0.5, 95\% CI $0.1-2.1, \mathrm{p}=0.4)$. Results were no different using the Barthel index (data not shown).

In a multivariate analysis including age, disease severity (PSI), ICU admission and functional status (ECOG), the latter was found to be independently associated with mortality (OR 4.0. 95\% CI 1.3-12.0. $\mathrm{p}=0.01$ ). When the above analysis was limited to only those patients with CAP $(n=397)$, functional status (ECOG) remained an independent prognostic factor (OR 4.6. 95\% CI 1.3-16.8. $\mathrm{p}=0.02$ ). 
Table 5. - Treatment given and outcome

\begin{tabular}{|c|c|c|c|c|}
\hline & NHAP & $\begin{array}{l}\text { CAP age } \\
\geqslant 65 \text { yrs }\end{array}$ & $\begin{array}{l}\text { All } \\
\text { CAP }\end{array}$ & p-value \\
\hline Subjects n & 40 & 236 & 397 & \\
\hline $\begin{array}{l}\text { Empirical } i . v . \\
\text { antibiotics }\end{array}$ & $37(93)^{*}$ & $176(75)^{*}$ & $294(74)$ & $0.03^{\#}$ \\
\hline "i.v. antibiotics days" & $3.11 \pm 3$ & $2.9 \pm 2.2$ & $3.2 \pm 2.6$ & \\
\hline $\begin{array}{l}\text { Median "i.v. } \\
\text { antibiotics days" }\end{array}$ & 2 & 2.3 & 2.7 & \\
\hline ICU admission $n$ & 0 & 8 & 20 & 0.10 \\
\hline ICU deaths & 0 & $4(50)$ & $4(20)$ & \\
\hline Died & $21(53)^{*}$ & $49(21)^{*}$ & $53(13)$ & $<0.001^{\#}$ \\
\hline $\begin{array}{l}\text { Mean LOS survivors } \\
\text { days }\end{array}$ & 11.2 & 10.8 & 9.1 & \\
\hline Median days & 6 & 8 & 7 & \\
\hline Geometric mean & 1.97 & 2.1 & 1.92 & 0.90 \\
\hline
\end{tabular}

Data are presented as mean \pm SD and $n(\%)$ unless otherwise stated. NHAP: nursing home acquired pneumonia; CAP: community acquired pneumonia; ICU: intensive care unit; LOS: length of stay; \#: age-adjusted: comparing NHAP with all CAP. One "i.v. antibiotics day" is equivalent to 1 day's equivalent i.v. doses, e.g. three doses i.v. cefuroxime, four doses i.v. erythromycin. *: statistically significant difference ( $\mathrm{p}<0.05$, not age-adjusted).

Comparing patients with nursing home acquired pneumonia and older patients with community acquired pneumonia

The subgroup of patients aged $\geqslant 65$ yrs with CAP $(n=236)$ were compared to patients with NHAP in separate analyses. Four patients with NHAP were aged $<65$ yrs although, there was no difference in the ages of the two groups. The major findings mirror those reported using the entire cohort details, are given in tables 1-3 and 5. Multivariate analysis including age ICU admission, disease severity and functional status confirmed the independent association of the latter with mortality (ECOG OR 3.6. 95\% CI 1.210.7, $\mathrm{p}=0.02$ ).

\section{Discussion}

This is the first report of NHAP in the UK. It shows that patients with NHAP have a high mortality, but that the aetiology of the infection is similar to patients admitted from the community.

NHAP was found to be relatively uncommon, accounting for only $9 \%$ of all the CAP patients admitted over a 2-yr period to one of two large hospitals sharing equally acute care for an urban population of 700,000 . However, the present study provides new information relevant to the UK and other similar healthcare systems, which is important in view of the high mortality $(53 \%)$ found in hospitalized patients.

\section{Presenting clinical features}

NHAP patients were more likely to present to hospital with mental confusion, low serum albumin and severe pneumonia, but less likely to present with a productive cough, dyspnoea and pleuritic chest pain compared to patients with CAP. The lower level of respiratory symptoms in NHAP patients was not evident after adjustment for mental confusion (data not shown), indicating that impaired recall of symptoms by the patient or staff in an institution, rather than when living at home with a member of the family, is the main explanation.

It is recognized that older patients with CAP present with less specific symptoms compared to younger patients. Lack of a febrile response, being "off legs" and confusion are all common modes of presentation in these older patients [16]. Studies of CAP in the elderly report the presence of mental confusion on admission in $17-45 \%$ of patients [6, 17-20], similar to that described for patients in longterm care facilities with pneumonia $(21-50 \%)[4,21$, 22]. MARrie and Blanchard [4] noted a nonsignificant trend towards more patients with NHAP displaying confusion compared to patients with CAP $(50 \%$ versus 35\%) [4]. This study extends and confirms this association.

The lower serum albumin among patients with NHAP is interesting. MARrie and Blanchard [4] made a similar observation, although serum albumin was measured in only $24 \%$ of NHAP patients in that study [4]. An increase in pro-inflammatory cytokines in response to acute infection is one explanation for the low albumin levels observed [23]. The extent to which the nutritional status of these patients prior to the onset of pneumonia contributed to the low albumin levels on admission is unknown. This may be important in view of evidence from epidemiological studies describing the association of lower serum albumin levels with limitations in activities of daily living, nursing home residence, poorer health and increased mortality [24, 25].

\section{Aetiology}

Evidence was found for S. pneumoniae infection in just under half of patients with NHAP, similar to patients admitted with CAP. Studies that have reported the aetiology of NHAP have varied greatly in the range of diagnostic tests employed and the predominant pathogens identified, with EGNB in $0-55 \%$ and $S$. aureus in $0-33 \%$ of patients. Adequate samples of sputum have generally been difficult to obtain; on average obtained in about one-third of patients [10]. Furthermore, sputum culture results are difficult to interpret in the presence of upper airways colonized with EGNB, which can occur in up to $50 \%$ of nursing home residents and may lead to overestimation of the contribution of EGNB to NHAP [26]. The sputum sampling rate in the present study was comparable to other studies. In addition, a combination of cultural, serological and antigen detection techniques were used and a pathogen was identified in $68 \%$ of cases and $80 \%$ of controls. Therefore, it is felt that the present results are likely to be representative. The finding of infection by C. pneumoniae in $18 \%$ of NHAP and $16 \%$ of CAP 
patients is consistent with other studies of adult CAP, where $C$. pneumoniae has been implicated in 10-18\% of cases $[27,28]$. This can be compared with a recent report from Canada of $C$. pneumoniae infection in $44-68 \%$ of nursing home residents, suggesting an endemic focus in that environment [29]. The increased rates of influenza infection among controls is difficult to explain as influenza immunization rates were similar in the two groups. It may relate to reduced exposure to potentially infected people in a nursing home, related to low visitor contact and an active vaccination policy for nursing home staff members. No data are available on this.

\section{Is nursing home acquired pneumonia associated with increased mortality?}

NHAP can be confirmed as an independent poor prognostic factor when adjusted for disease severity, but not when adjusted for functional status. This is an important distinction and suggests that poor functional status is the main factor that explains the higher mortality in NHAP compared to CAP. It may be argued that the poorer premorbid functional status of patients with NHAP negatively influenced ICU admission decisions and led to the increased mortality observed in this group. Adjustment for ICU admission was, therefore, included in the multivariate analysis. Even allowing for an ICU admission rate of $10 \%$ in NHAP patients and a conservative estimate of only $20 \%$ ICU mortality in these patients, overall mortality in NHAP would still have been $45 \%$ (18 patients). Therefore, it is unlikely that differences in ICU admission rates alone account for the findings. When measured, functional status has consistently been shown to be associated with both short-term and long-term mortality in studies conducted in long-term care facilities $[4,9]$. The presented data suggest that functional status is an important independent predictor of pneumonia mortality, in addition to disease severity regardless of nursing home residence status. Cognitive impairment, poor functional status, nursing home residence and increased mortality from pneumonia are all interrelated [30].

\section{Implications for management}

These data suggest that the choice of empirical antibiotics in patients admitted to hospital with NHAP in the UK should not be different than for older patients with CAP. Functional status appears important as an additional risk factor for outcome in pneumonia. It is usually well assessed in patients admitted to hospital with cerebrovascular disease including stroke, but is not recognized as an important measure of disease severity in patients admitted with CAP. Increased appreciation of the importance of recording functional status and mental confusion as part of severity assessment in CAP, especially in patients admitted from nursing homes, is necessary.

\section{Study limitations}

This study was hospital based and it is likely that nursing home residents seen and managed by their General Practitioner were missed, as they were never referred to hospital. The proportion of patients referred to hospital from published series varies from $9-51 \%$ and is dependent on a variety of clinical and nonclinical factors [10]. In general, patients who are hospitalized are more severely ill. However, nursing home patients who are most ill and who are judged to be unlikely to benefit from more aggressive treatment, may not be referred to hospital. In the aetiological arm of the present study, detailed microbiological tests were performed in only $22(60 \%)$ of patients with NHAP. Therefore, small differences in the frequencies of pathogens implicated in NHAP compared with CAP would not have been detected and the possibility that the lack of EGNB identified may have been due to inadequate sampling cannot be dismissed. The spectrum of pathogens described here for NHAP may, therefore, best be considered preliminary and awaits confirmation within a larger study cohort.

\section{Conclusions}

Patients hospitalized with nursing home acquired pneumonia are less likely to present with respiratory symptoms, but are more likely to display mental confusion than patients with community acquired pneumonia. Patients with nursing home acquired pneumonia have clinically more severe disease compared to patients with community acquired pneumonia. The pathogens implicated are similar. The increased mortality associated with nursing home acquired pneumonia compared to community acquired pneumonia is explained mainly by differences in premorbid functional status, which is an important prognostic factor that should be assessed in all patients admitted to hospital with pneumonia.

\footnotetext{
Acknowledgements. The authors would like to thank Binax for donating the Streptococcus pneumoniae Binax-NOW antigen detection kits. The authors are also especially grateful to: T. Boswell, R. Cave, J. Palfryman and the team of technical and medical staff at Nottingham Public Health Laboratory for their dedication to the study; M. Leinonen, P. Saikku and A. Jaakkola at the National Public Health Institute, Dept in Oulu, Finland; T. Harrison at the Central Public Health Laboratory, Colindale; D. Rose for expert radiological support and physicians at Nottingham City Hospital for allowing us to study their patients.
}

\section{References}

1. Gross JS, Neufeld RR, Libow LS, Gerber I, Rodstein M. Autopsy study of the elderly institutionalized patient. Review of 234 autopsies. Arch Intern Med 1988; 148: 173-176. 
2. Kerr HD, Byrd JC. Nursing home patients transferred by ambulance to a VA emergency department. $\mathrm{J} \mathrm{Am}$ Genatr Soc 1991; 39: 132-136.

3. Marrie TJ, Durant H, Yates L. Community-acquired pneumonia requiring hospitalization: 5-year prospective study. Rev Infect Dis 1989; 11: 586-599.

4. Marrie TJ, Blanchard W. A comparison of nursing home-acquired pneumonia patients with patients with community-acquired pneumonia and nursing home patients without pneumonia. J Am Geriatr Soc 1997; 45: 50-55.

5. Garb JL, Brown RB, Garb JR, Tuthill RW. Differences in etiology of pneumonias in nursing home and community patients. JAMA 1978; 240: 2169-2172.

6. Janssens JP, Gauthey L, Herrmann F, Tkatch L, Michel JP. Community-acquired pneumonia in older patients. J Am Genatr Soc 1996; 44: 539-544.

7. Meehan TP, Chua-Reyes JM, Tate J, et al. Process of care performance, patient characteristics, and outcomes in elderly patients hospitalized with community-acquired or nursing home-acquired pneumonia. Chest 2000; 117: 1378-1385.

8. Naughton BJ, Mylotte JM. Treatment guideline for nursing home-acquired pneumonia based on community practice. J Am Genatr Soc 2000; 48: 82-88.

9. Muder RR, Brennen C, Swenson DL, Wagener M. Pneumonia in a long-term care facility. A prospective study of outcome. Arch Intern Med 1996; 156: 2365 2370.

10. Muder RR. Pneumonia in residents of long-term care facilities: epidemiology, etiology, management, and prevention. Am J Med 1998; 105: 319-330.

11. Fried TR, Gillick MR, Lipsitz LA. Whether to transfer? Factors associated with hospitalization and outcome of elderly long-term care patients with pneumonia. J Gen Intern Med 1995; 10: 246-250.

12. Neill AM, Martin IR, Weir R, et al. Community acquired pneumonia: aetiology and usefulness of severity criteria on admission. Thorax 1996; 51: 1010-1016.

13. Fine MJ, Auble TE, Yealy DM, et al. A prediction rule to identify low-risk patients with communityacquired pneumonia. N Engl J Med 1997; 336: 243250.

14. Lim WS, Macfarlane JT, Boswell TC, et al. SCAPA. Study of community acquired pneumonia aetiology (SCAPA) in adults admitted to hospital: Implications for management guidelines. Thorax 2001; 56: 296-301.

15. Gladman J, Barer D, Venkatesan P, Berman P, Macfarlane JT. The outcome of pneumonia in the elderly: a hospital survey. Clin Rehabil 1991; 5: 201205.

16. Lieberman D, Schlaeffer F, Porath A. Communityacquired pneumonia in old age: a prospective study of
91 patients admitted from home. Age Ageing 1997; 26: 69-75.

17. Riquelme R, Torres A, El-Ebiary M, et al. Community-acquired pneumonia in the elderly: A multivariate analysis of risk and prognostic factors. Am J Respir Crit Care Med 1996; 154: 1450-1455.

18. Venkatesan P, Gladman J, Macfarlane JT, et al. A hospital study of community acquired pneumonia in the elderly. Thorax 1990; 45: 254-258.

19. Starczewski AR, Allen SC, Varga E, Lye M. Clinical prognostic indices of fatality in elderly patients admitted to hospital with acute pneumonia. Age Ageing 1988; 17: 181-186.

20. Lim WS, Macfarlane JT. Defining prognostic factors in the elderly with community acquired pneumonia: a case controlled study of patients aged $\geqslant 75$ yrs. Eur Respir J 2001; 17: 200-205.

21. Marrie TJ, Durant H, Kwan C. Nursing homeacquired pneumonia. A case-control study. $J \mathrm{Am}$ Geriatr Soc 1986; 34: 697-702.

22. Peterson PK, Stein D, Guay DR, et al. Prospective study of lower respiratory tract infections in an extended-care nursing home program: potential role of oral ciprofloxacin. Am J Med 1988; 85: 164-171.

23. Hedlund JU, Hansson LO, Ortqvist AB. Hypoalbuminemia in hospitalized patients with communityacquired pneumonia. Arch Int Med 1995; 155: 14381442.

24. Salive ME, Cornoni-Huntley J, Phillips CL, et al. Serum albumin in older persons: relationship with age and health status. J Clin Epidemiol 1992; 45: 213-221.

25. Klonoff-Cohen H, Barrett-Cor nor EL, Edelstein SL. Albumin levels as a predictor of mortality in the healthy elderly. J Clin Epidemiol 1992; 45: 207-212.

26. Valenti WM, Trudell RG, Bentley DW. Factors predisposing to oropharyngeal colonization with gram-negative bacilli in the aged. $N$ Engl $J \mathrm{Med}$ 1978; 298: 1108-1011.

27. Lieberman D, Schlaeffer F, Boldur I, et al. Multiple pathogens in adult patients admitted with communityacquired pneumonia: a one year prospective study of 346 consecutive patients. Thorax 1996; 51: 179-184.

28. Steinhoff D, Lode H, Ruckdeschel G, et al. Chlamydia pneumoniae as a cause of community-acquired pneumonia in hospitalized patients in Berlin. Clin Infect Dis 1996; 22: 958-964.

29. Troy CJ, Peeling RW, Ellis AG, et al. Chlamydia pneumoniae as a new source of infectious outbreaks in nursing homes (published erratum appears in $J A M A$ 1997; 278: 118). JAMA 1997; 277: 1214-1218.

30. Salive ME, Satterfield S, Ostfeld AM, Wallace RB, Havlik RJ. Disability and cognitive impairment are risk factors for pneumonia-related mortality in older adults. Public Health Rep 1993; 108: 314-322. 